01.5

\title{
Метод определения характеристик перемежающейся обобщенной синхронизации, основанный на вычислении вероятности наблюдения синхронного режима
}

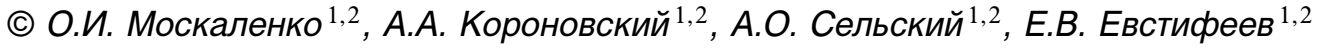 \\ ${ }^{1}$ Саратовский национальный исследовательский государственный университет им. Н.Г. Чернышевского, Саратов, Россия \\ ${ }^{2}$ Региональный научно-образовательный математический центр „Математика технологий будущего“, Саратов, Россия \\ E-mail: o.i.moskalenko@gmail.com
}

Поступило в Редакцию 5 августа 2021 г.

В окончательной редакции 5 августа 2021 г.

Принято к публикации 21 сентября 2021 г.

Предложен метод выделения характерных фаз поведения однонаправленно связанных систем, находящихся вблизи границы возникновения режима обобщенной хаотической синхронизации, основанный на вычислении вероятности наблюдения синхронного режима по ансамблю связанных систем. На примере однонаправленно связанных систем Ресслера, находящихся в режиме ленточного хаоса, показана его эффективность по сравнению с другими известными методами определения характеристик перемежающейся обобщенной синхронизации.

Ключевые слова: обобщенная синхронизация, перемежающееся поведение, однонаправленно связанные системы, мультистабильность, ламинарные фазы поведения, вероятность наблюдения ламинарной фазы.

DOI: 10.21883/PJTF.2022.02.51910.18985

Перемежающееся поведение характерно для систем различной природы и представляет собой универсальное явление [1]. Перемежаемость является одним из классических сценариев при переходе от периодических колебаний к хаотическим, а также наблюдается вблизи границ синхронных режимов, в связи с чем выделяют перемежающуюся полную синхронизацию, перемежающуюся синхронизацию с запаздыванием, перемежающуюся обобщенную синхронизацию, перемежающуюся индуцированную шумом синхронизацию, перемежающуюся фазовую синхронизацию и перемежающуюся синхронизацию временны́х масштабов [2-9].

Каждый из этих типов перемежающегося синхронного поведения имеет свои механизмы возникновения и свои характеристики длительностей ламинарных (синхронных) фаз. Более того, в ряде случаев тип перемежаемости зависит не только от типа синхронизации, но и от величины расстройки между взаимодействующими системами или топологии аттракторов этих систем. Так, например, вблизи границы фазовой синхронизации в случае относительно слабой расстройки между системами наблюдается перемежаемость „игольного ушка“, а при большой расстройке - перемежаемость „кольца“ $[6,7]$. На границе обобщенной синхронизации в системах с относительно простой топологией аттрактора имеет место перемежаемость типа „оn-off“ [3], в то время как в системах с относительно сложной (двулистной) структурой наблюдается перемежаемость перескоков [9]. Механизмы возникновения всех названных выше типов перемежаемости и статистические характеристики оказываются также различными.
Для определения механизмов возникновения перемежаемости и расчета статистических характеристик перемежающегося поведения важную роль играют методы выделения характерных фаз динамики систем. Для режима перемежающейся обобщенной синхронизации известно несколько таких методов, основанных на расчете локальных показателей Ляпунова [4], применении непрерывного вейвлетного преобразования [10] или анализе расположения изображающих точек на аттракторах взаимодействующих систем [9]. В то же время самым распространенным и эффективным методом анализа перемежающейся обобщенной синхронизации является метод вспомогательной системы [11]. Этот метод может быть легко применен к однонаправленно связанным динамическим системам, тогда как для систем со взаимной связью он приводит к некорректным результатам [12].

Метод вспомогательной системы [11] сводится к следующему: наряду с ведомой системой рассматривается идентичная ей вспомогательная система, находящаяся под воздействием той же самой ведущей системы. Начальные условия для вспомогательной системы должны отличаться от начального состояния ведомой системы, но лежать в бассейне притяжения того же самого хаотического аттрактора. В режиме обобщенной синхронизации между состояниями ведущей и ведомой, а также ведущей и вспомогательной систем устанавливается функциональная связь (функционал) [13,14], что приводит к идентичности состояний ведомой и вспомогательной систем после завершения переходного процесса. В отсутствие обобщенной синхронизации состояния ведомой и вспомогательной систем оказы- 
ваются всегда различными, в то время как в режиме перемежающейся обобщенной синхронизации состояния ведомой и вспомогательной систем совпадают друг с другом только на определенных интервалах времени, называемых ламинарными (синхронными) фазами, а в остальные моменты времени реализуются фазы асинхронного поведения (турбулентные всплески) [3].

Для определения характеристик перемежаемости необходимо проанализировать сигнал, представляющий собой временну́ю зависимость разности состояний ведомой и вспомогательной систем [3]. Те интервалы времени, где модуль этой разности будет меньше некоторой наперед заданной малой величины $\Delta$, соответствуют ламинарным фазам поведения, остальные моменты времени - турбулентным фазам. По статистике длительностей ламинарных фаз (распределениям длительностей ламинарных фаз при фиксированных значениях управляющих параметров и зависимости средней длительности ламинарных фаз от параметра связи) можно однозначно определить тип перемежаемости, реализующийся в системе.

При анализе перемежающегося поведения вблизи границы обобщенной синхронизации при помощи метода вспомогательной системы важную роль играет вопрос о выборе начальных условий для ведомой и вспомогательной систем. Необходимо отметить, что до сих пор этот вопрос в литературе, как правило, не обсуждался (за исключением того, что начальные условия для этих систем должны быть различными, но принадлежащими одному и тому же бассейну притяжения). Как показано далее, выбор начальных условий для этих систем оказывается весьма существенным для диагностируемого в фиксируемый момент времени режима. Иными словами, в режиме перемежающейся обобщенной синхронизации однонаправленно связанных систем при одном и том же состоянии ведущей системы в зависимости от начальных условий ведомой может диагностироваться как синхронная, так и асинхронная динамика, т.е. имеет место мультистабильность.

Наличие мультистабильности вблизи границы обобщенной синхронизации ставит вопрос о совершенствовании существующих методов выделения характерных фаз поведения систем с учетом этой особенности. В настоящей работе предложен метод выделения ламинарных фаз вблизи границы обобщенной синхронизации, основанный на вычислении вероятности наблюдения синхронного режима по ансамблю связанных систем. Метод основан на рассмотрении ведущего осциллятора и относительно большого ансамбля однонаправленно связанных с ним ведомых систем и вычислении вероятности наблюдения ламинарной фазы поведения (синхронного участка поведения) в зависимости от времени.

В качестве объекта исследования выбран ансамбль однонаправленно связанных слабо неидентичных систем
Ресслера, находящихся в режиме ленточного хаоса:

$$
\begin{gathered}
\dot{x}_{d}=-\omega_{d} y_{d}-z_{d}, \\
\dot{y}_{d}=\omega_{d} x_{d}+a y_{d}, \\
\dot{z}_{d}=p+z_{d}\left(x_{d}-c\right), \\
\dot{x}_{r}^{i}=-\omega_{r} y_{r}^{i}-z_{r}^{i}+\varepsilon\left(x_{d}-x_{r}^{i}\right), \\
\dot{y}_{r}^{i}=\omega_{r} x_{r}^{i}+a y_{r}^{i}, \\
\dot{z}_{r}^{i}=p+z_{r}^{i}\left(x_{r}^{i}-c\right),
\end{gathered}
$$

где индексы $d$ и $r$ относятся к ведущей и ведомым системам соответственно, индекс $i=1, \ldots, N$ соответствует номеру ведомой системы, начальные условия для этих систем выбираются различными, $a=0.15$, $p=0.2, c=10, \omega_{d}=0.93$ и $\omega_{r}=0.95$ выступают в роли управляющих параметров взаимодействующих систем, $\varepsilon$ - параметр связи. При выбранных значениях управляющих параметров и $\varepsilon \geqslant 0.178$ в системе (1) наблюдается режим обобщенной синхронизации [15]. Вблизи границы синхронного режима, как обсуждалось выше, имеет место перемежающееся поведение.

Для выделения характерных фаз поведения в системе (1) и определения характеристик перемежаемости введем в рассмотрение вероятность наблюдения турбулентной фазы

$$
P_{a}=1-\sum_{i=1}^{N} \frac{n\left(\mathbf{x}_{r}^{i}\right)}{N(N-1)}
$$

(где $n\left(\mathbf{x}_{r}^{i}\right)$ - число систем, находящихся в синхронном режиме с $i$-м осциллятором, $N=1000$ - число осцилляторов в ансамбле) и рассмотрим ее динамику с течением времени. Если $P_{a}$ близка к единице, в исследуемой системе наблюдается турбулентная фаза поведения. При значениях $P_{a}$, близких к нулю, диагностируется ламинарная фаза. Понятно, что $P_{a}$ может принимать промежуточные значения в диапазоне $(0,1)$. Тогда, задав порог разделения ламинарных и турбулентных фаз, можно разделить характерные фазы поведения взаимодействующих систем.

На рис. 1, $a$ приведена зависимость $P_{a}(t)$ для системы (1) при $\varepsilon=0.17$. На рис. $1, b$ показана разность между состояниями двух ведомых (одна из которых играет роль вспомогательной) систем $\xi(t)=\left|x_{r}^{1}-x_{r}^{2}\right|$ при том же значении параметра связи. Из сопоставления рис. $1, a$ и $b$ видно, что характерные фазы поведения систем, выделенные двумя различными способами, несколько отличаются друг от друга, хотя и оказываются сосредоточенными вблизи одних и тех же временны́х интервалов. Более того, при определении длительностей ламинарных фаз по вероятности детектирования турбулентной фазы переход от асинхронного состояния к синхронному сопровождается достаточно резким ростом вероятности, что позволяет избежать детектирования ультракоротких ламинарных фаз (что типично для метода вспомогательной системы) и, следовательно, 

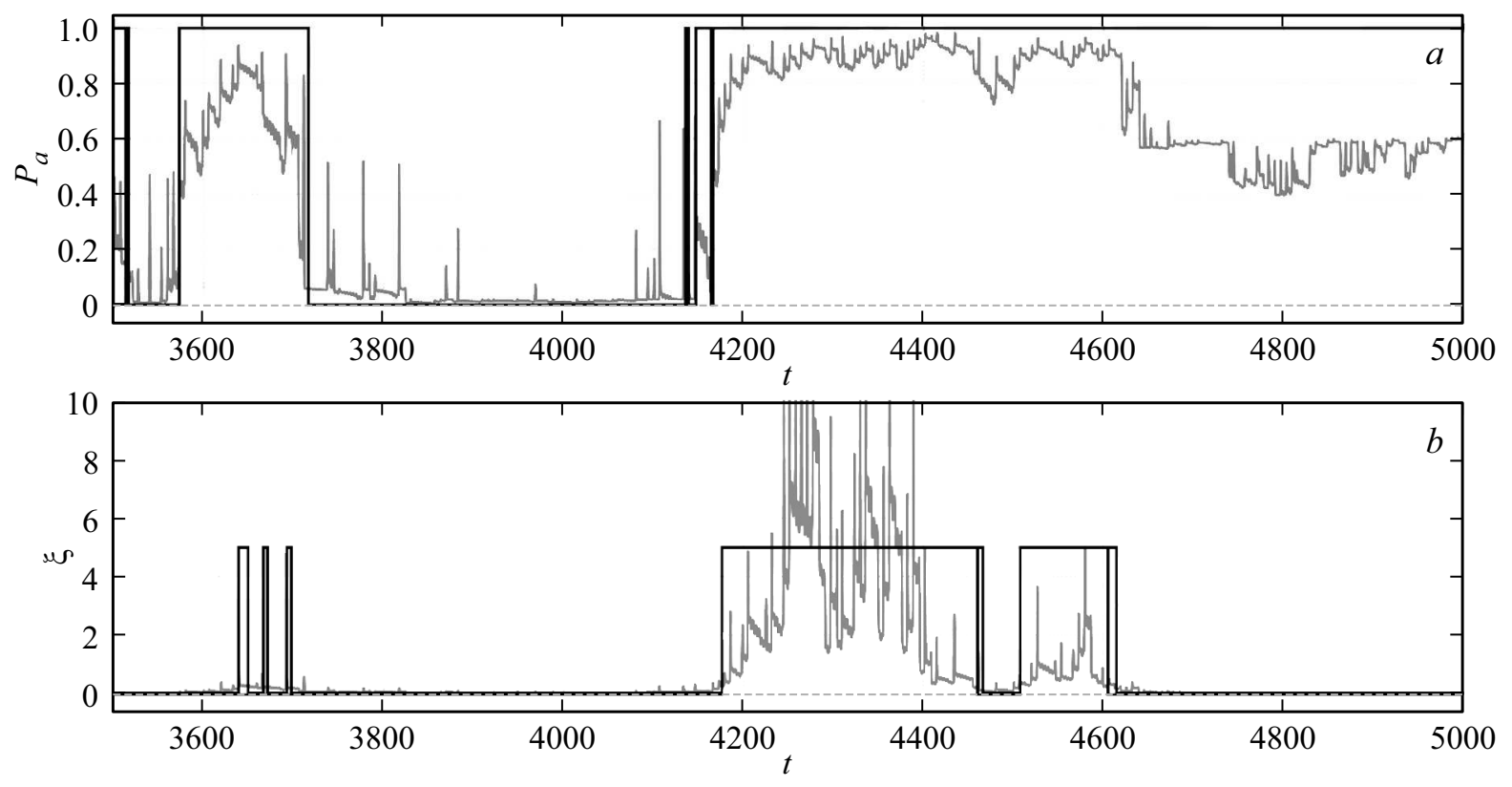

Рис. 1. Временна́я зависимость вероятности наблюдения фазы асинхронного поведения $P_{a}$ для ансамбля однонаправленно связанных систем Ресслера $(1)$ при $\varepsilon=0.17(a)$ и аналогичная зависимость разности между состояниями двух ведомых (ведомой и вспомогательной) систем $\xi(t)=\left|x_{r}^{1}-x_{r}^{2}\right|$ при том же значении параметра связи $(b)$.

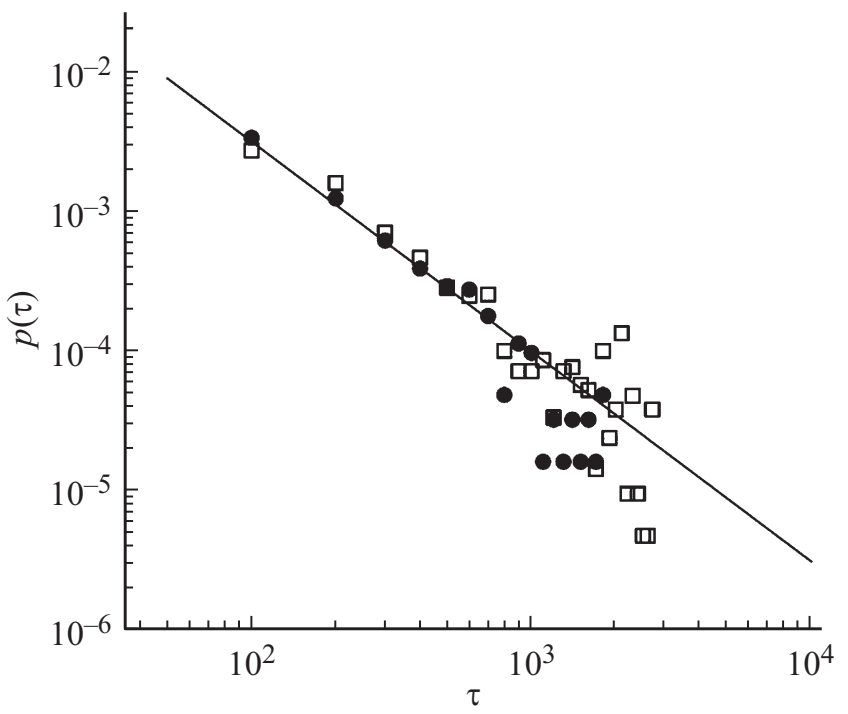

Рис. 2. Распределения длительностей ламинарных фаз однонаправленно связанных систем Ресслера (1), полученные при $\varepsilon=0.17$ при помощи двух методов: метода вспомогательной системы (квадраты) и вероятностного метода, предложенного в настоящей работе (кружки), а также их теоретическая аппроксимация степенным законом (3), $k=3$. Среднеквадратичные отклонения данных численного моделирования от теоретической закономерности составляют $\sigma_{1}=4.9 \cdot 10^{-9}$ и $\sigma_{2}=1.6 \cdot 10^{-9}$ соответственно.

обеспечивает более точное определение длительностей характерных фаз поведения систем.

На рис. 2 приведена одна из статистических характеристик длительностей ламинарных фаз - распределе- ния длительностей ламинарных фаз, рассчитанные при значении параметра связи $\varepsilon=0.17$ двумя различными способами: методом вспомогательной системы и по вероятности, а также их теоретическая аппроксимация степенным законом

$$
p(\tau)=k \tau^{-3 / 2}
$$

(где $k-$ положительная константа), характерным для „on-off“ перемежаемости [3]. Видно, что в обоих случаях данные численного моделирования хорошо аппроксимируются степенным законом (3), но среднеквадратичное отклонение данных численного моделирования от теоретической закономерности при применении предложенного метода оказывается значительно меньше (см. подпись к рис. 2). Отмеченное выше указывает на возможность применения предложенного подхода для определения характеристик перемежаемости в более сложных системax, когда традиционными методами невозможно однозначно определить, степенной или экспоненциальный закон наблюдается в данном случае, что открывает широкие возможности применения этого метода на практике.

\section{Финансирование работы}

Работа выполнена при финансовой поддержке Российского научного фонда (проект № 19-12-00037). Получение статистических характеристик длительностей ламинарных фаз при помощи метода вспомогательной системы осуществлялось при поддержке Совета по грантам Президента РФ для государственной поддержки молодых ученых — докторов наук (проект № МД-21.2020.2). 


\section{Конфликт интересов}

Авторы заявляют, что у них нет конфликта интересов.

\section{Список литературы}

[1] П. Берже, И. Помо, К. Видаль, Порядок в хаосе (Мир, M., 1991).

[2] S. Boccaletti, D.L. Valladares, Phys. Rev. E, 62 (5), 7497 (2000). DOI: 10.1103/PhysRevE.62.7497

[3] A.E. Hramov, A.A. Koronovskii, Europhys. Lett., 70 (2), 169 (2005). DOI: $10.1209 / \mathrm{epl} / \mathrm{i} 2004-10488-6$

[4] О.И. Москаленко, Е.В. Евстифеев, А.А. Короновский, Письма в ЖТФ, 46 (16), 12 (2020).

DOI: $10.21883 /$ PJTF.2020.16.49846.18359

[5] О.И. Москаленко, А.А. Короновский, С.А. Шурыгина, ЖТФ, 81 (9), 150 (2011).

[6] A.S. Pikovsky, G.V. Osipov, M.G. Rosenblum, M. Zaks, J. Kurths, Phys. Rev. Lett., 79 (1), 47 (1997). DOI: 10.1103/PhysRevLett.79.47.

[7] A.E. Hramov, A.A. Koronovskii, M.K. Kurovskaya, S. Boccaletti, Phys. Rev. Lett., 97 (11), 114101 (2006). DOI: 10.1103/PhysRevLett.97.114101

[8] М.О. Журавлев, А.А. Короновский, О.И. Москаленко, А.Е. Храмов, ЖТФ, 81 (7), 7 (2011).

[9] A.A. Koronovskii, O.I. Moskalenko, A.A. Pivovarov, V.A. Khanadeev, A.E. Hramov, A.N. Pisarchik, Phys. Rev. E, 102 (1), 012205 (2020). DOI: 10.1103/PhysRevE.102.012205

[10] Е.В. Евстифеев, О.И. Москаленко, Изв. РАН. Сер. физ., 84 (2), 300 (2020). DOI: 10.31857/S0367676520020106

[11] H.D.I. Abarbanel, N.F. Rulkov, M. Sushchik, Phys. Rev. E, 53 (5), 4528 (1996). DOI: 10.1103/PhysRevE.53.4528

[12] O.I. Moskalenko, A.A. Koronovskii, A.E. Hramov, Phys. Rev. E, 87 (6), 064901 (2013). DOI: 10.1103/PhysRevE.87.064901

[13] N.F. Rulkov, M.M. Sushchik, L.S. Tsimring, H.D.I. Abarbanel, Phys. Rev. E, 51 (2), 980 (1995). DOI: 10.1103/PhysRevE.51.980

[14] A.A. Koronovskii, O.I. Moskalenko, A.E. Hramov, Phys. Rev. E, 84 (3), 037201 (2011). DOI: 10.1103/PhysRevE.84.037201

[15] A.E. Hramov, A.A. Koronovskii, O.I. Moskalenko, Europhys. Lett., 72 (6), 901 (2005). DOI: 10.1209/ep1/i2005-10343-4 